\title{
Resection outcomes for primary and local recurrent retroperitoneal liposarcoma patients
}

\author{
Yang Yan ${ }^{1 \#}$, Shaoyou Xia ${ }^{1 \#}$, Da Teng ${ }^{1}$, Shidong $\mathrm{Hu}^{1}$, Songyan $\mathrm{Li}^{1}$, Yufeng Wang ${ }^{2}$, Xiaohui $\mathrm{Du}^{1}$, Rong $\mathrm{Li}^{1}$ \\ ${ }^{1}$ Department of General Surgery, ${ }^{2}$ Department of Patient Admission Management, the First Medical Center, Chinese PLA General Hospital, \\ Beijing, China \\ Contributions: (I) Conception and design: Y Yan, X Du; (II) Administrative support: S Xia, D Teng; (III) Provision of study materials or patients: S Li, \\ Y Wang; (IV) Collection and assembly of data: Y Yan, S Xia, D Teng; (V) Data analysis and interpretation: Y Yan, S Hu, D Teng; (VI) Manuscript \\ writing: All authors; (VII) Final approval of manuscript: All authors. \\ \#These authors contributed equally to this work. \\ Correspondence to: Professor Xiaohui Du. General Surgery Department, First Medical Center, Chinese PLA General Hospital, 28 Fuxing Road, \\ Haidian, Beijing 100853, China. Email: duxiaohui5555@126.com.
}

Background: The clinical characteristics of primary retroperitoneal liposarcoma (PR RPLPS) and local recurrent retroperitoneal liposarcoma (LR RPLPS) cases were compared to determine the related factors involved in postoperative survival.

Methods: A total of 90 patients who underwent surgery between 2006 and 2013 were included in this study. Clinicopathological data that was prospectively gathered was analyzed to identify factors associated with overall survival (OS) and progression-free survival (PFS).

Results: The PR cases showed a higher complete resection rate when compared to the LR group. The LR group showed a greater number that were poorly differentiated and highly malignant. More blood loss was observed in the LR compared to the PR group. Multivariate analysis suggested that blood loss and tumor grade were prognostic factors for OS and PFS of the PR group, but extent of resection was a prognostic factor only for OS. In the LR group, the extent of resection was a significant prognostic factor associated with OS, whereas tumor grade was associated with PFS.

Conclusions: Complete surgical resection is the most important factor for the survival of RPLPS patients. Tumor grade is an independent prognostic factor for PFS. In PR RPLPS, poor tumor classification and increased intraoperative bleeding are associated with a poor prognosis.

Keywords: Retroperitoneal liposarcoma (RPLPS); primary tumor; local recurrence; retroperitoneal sarcoma; overall survival (OS); progression-free survival (PFS)

Submitted Aug10, 2020. Accepted for publication Oct 12, 2020.

doi: $10.21037 / \mathrm{atm}-20-6316$

View this article at: http://dx.doi.org/10.21037/atm-20-6316

\section{Introduction}

Retroperitoneal liposarcoma (RPLPS) is the most common retroperitoneal soft tissue sarcoma (RSTS), accounting for $20 \%$ of all soft tissue tumors (1-3). Primary RPLPS is a rare disease and surgery is the main mode of treatment. Recurrence of the tumor is the main impending issue, ultimately resulting in death. Local recurrence occurs in $70 \%$ of cases without exhibiting distant metastases (2).
Although strategies to combat local recurrence are critical, the understanding of the cause of the primary disease still needs to be investigated and fully understood.

Here, patients undergoing primary and secondary treatment for RPLPS were analyzed to explore factors affecting the prognosis of this disease from multiple dimensions. We present the following article in accordance with the STROBE reporting checklist (available at http:// dx.doi.org/10.21037/atm-20-6316). 


\section{Methods}

A total of 90 RPLPS patients undergoing surgical treatment at the PLA General Hospital (Beijing, China) from December 1, 2006 to June 1, 2013 were analyzed in this study. There were 46 cases of primary RPLPS, and 44 cases showing their first local recurrence. All cases were confirmed by the pathology department in the hospital. The study was conducted in accordance with the Declaration of Helsinki (as revised in 2013). This study was approved by the Protection of Human Subjects Committee of the Chinese People's Liberation Army (PLA) General Hospital. Informed written consent was provided by all participants included in the study.

Patient data included age, gender, tumor size, pathological classification, surgical method, intraoperative blood loss, adjuvant therapy, combined organ resection, and other pathological factors. All patients underwent computed tomography (CT), medical resonance imaging (MRI), or positron emission tomography (PET)/CT. For cases where imaging diagnosis was difficult, a preoperative biopsy was performed to remove all visible tumor. A negative resection margin was defined as $\mathrm{R} 0$ resection and a positive postoperative margin was considered a $\mathrm{R} 1$ resection [positive or close margins ( $<1 \mathrm{~mm}$ without intact fascia)]. If the tumor had invaded surrounding organs, combined organ resection was selected. However, if the tumor had invaded the blood vessels, a combined resection or ligation of the blood vessels was performed. A R2 (palliative) was considered if there was residual tumor observed during an operation.

Tumor size was expressed as the largest tumor diameter after a postoperative assessment (palliative resection cases). Tissue pathology was classified into grades 1, 2, and 3 according to the French Federation of Cancer Centres Sarcoma Group Grading System (Fédération Nationale des Centres de Lutte Contre le Cancer, FNCLCC). These grades were based on tumor cell morphology, degree of differentiation, heteromorphism, mitotic images, and necrosis. Adjuvant treatments included postoperative chemotherapy, intraoperative or postoperative radiotherapy, ultrasound-guided radiofrequency ablation, and biological therapy.

All patients participated in follow-ups every 3-4 months for 1 year following surgery. After 1 year, these follow-ups were performed every 6 months for 2-5 years post-surgery, and every year beyond the 5 years post-surgery date. Death of the patient, or the follow-up date 1 January 2020 was the endpoint of the analysis. Follow-up visits included surveillance imaging (CT chest/abdomen/pelvis). If tumor recurrence was noted, a shortened follow-up interval of 3-4 months was performed. Overall survival (OS) was defined as the time from initial presentation of disease until death. Progression-free survival (PFS) was defined as the time from diagnosis to the first occurrence of disease progression or death (date of first recurrence for those who had an R0/R1 resection, date of progression of residual disease for those who had R2 resection, or death without evidence of recurrence or progression).

\section{Statistical analysis}

SPSS version 22.0 (IBM, Armonk, NY, USA) statistical software was used for data analysis. Clinical data of the PR and LR groups were compared using the Pearson's chisquared $\left(\chi^{2}\right)$ or Wilcoxon-Mann-Whitney tests. The curves for OS and PFS were generated using the Kaplan-Meier method. Univariable and multivariable prognostic analyses were performed for OS and PFS to identify significant prognostic factors. Differences were considered statistically significant when $\mathrm{P}<0.05$.

\section{Results}

\section{Clinicopathologic characteristics}

Among the 90 cases of RPLPS treated, 46 cases were diagnosed as primary retroperitoneal liposarcoma (PR RPLPS) and 44 cases were diagnosed as local recurrent retroperitoneal liposarcoma (LR RPLPS). Patient demographics and tumor characteristics are listed in Table 1. The median age of patients was 54 years (30-87 years), and there was a slightly higher population of men $(58.9 \%)$ compared to women. The median tumor diameter was $19.5 \mathrm{~cm}(5-80 \mathrm{~cm})$. All cases were classified according to the FNCLCC criteria. Compared to the PR group $(41.3 \%$ of grade $1 ; 21.7 \%$ of grade $2 ; 37.0 \%$ of grade 3 ), there was an increased number of poorly differentiated and highly malignant cases in the LR group (18.2\% of grade $1 ; 22.7 \%$ of grade $2 ; 59.1 \%$ of grade $3, \mathrm{P}=0.042$ ).

\section{Treatment strategy}

A total of $51.1 \%(46 / 90)$ of patients underwent R0 resection, 33.3\% (30/90) underwent $\mathrm{R} 1$ resection, and $15.6 \%$ (14/90) underwent R2 resection. The primary group (R0, 67.4\%; R1, 26.1\%; R2, 6.5\%) showed a higher 
Table 1 Clinicopathological factors for 90 RPLPS patients

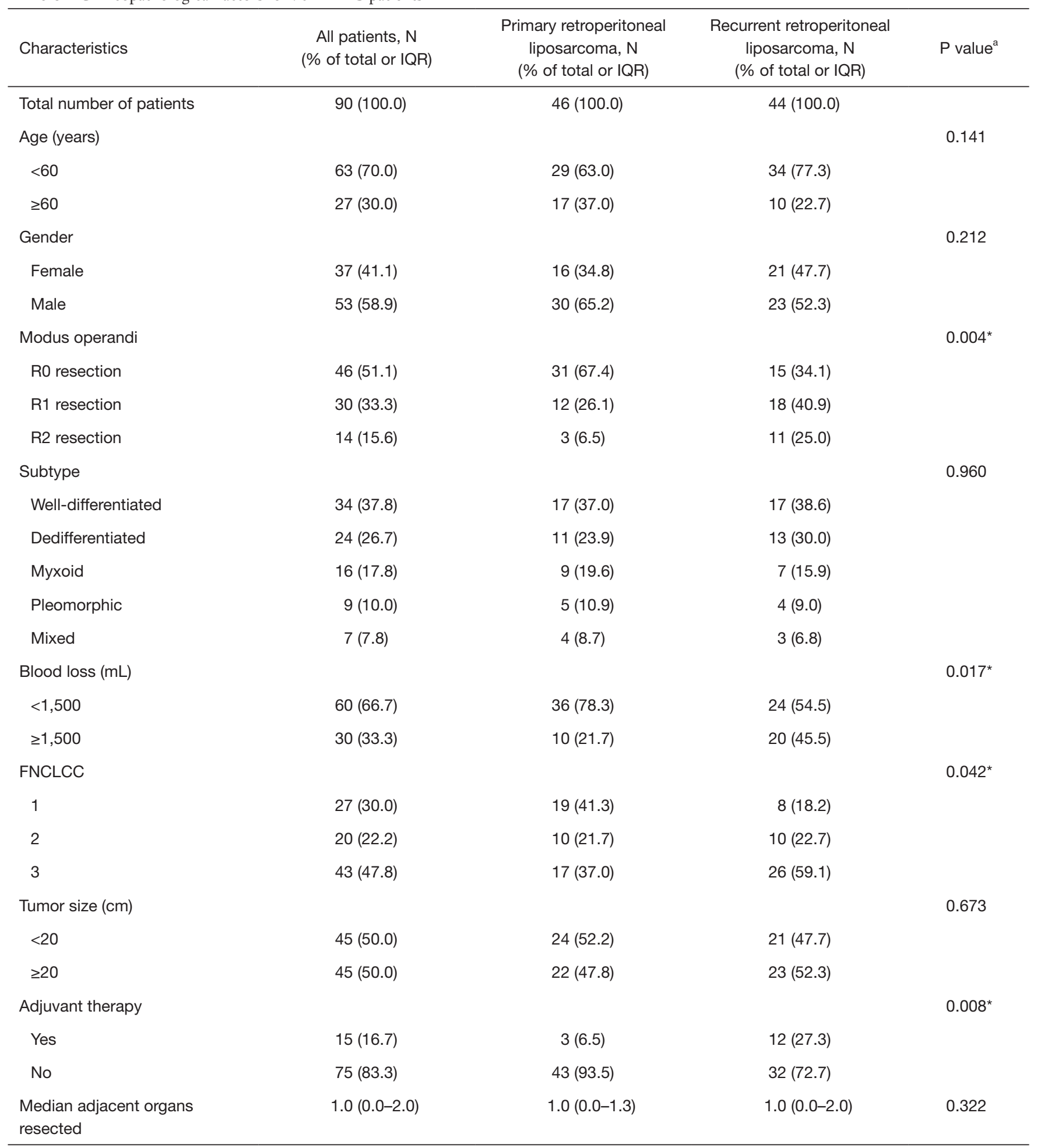

*, significant. ${ }^{a}$, comparison between patients who were primary retroperitoneal liposarcoma and patients who were recurrent retroperitoneal liposarcoma using Pearson's chi-squared test for categorical variables and Wilcoxon-Mann-Whitney test for continuous variables. IQR, inter-quartile range; RPLPS, retroperitoneal liposarcoma 

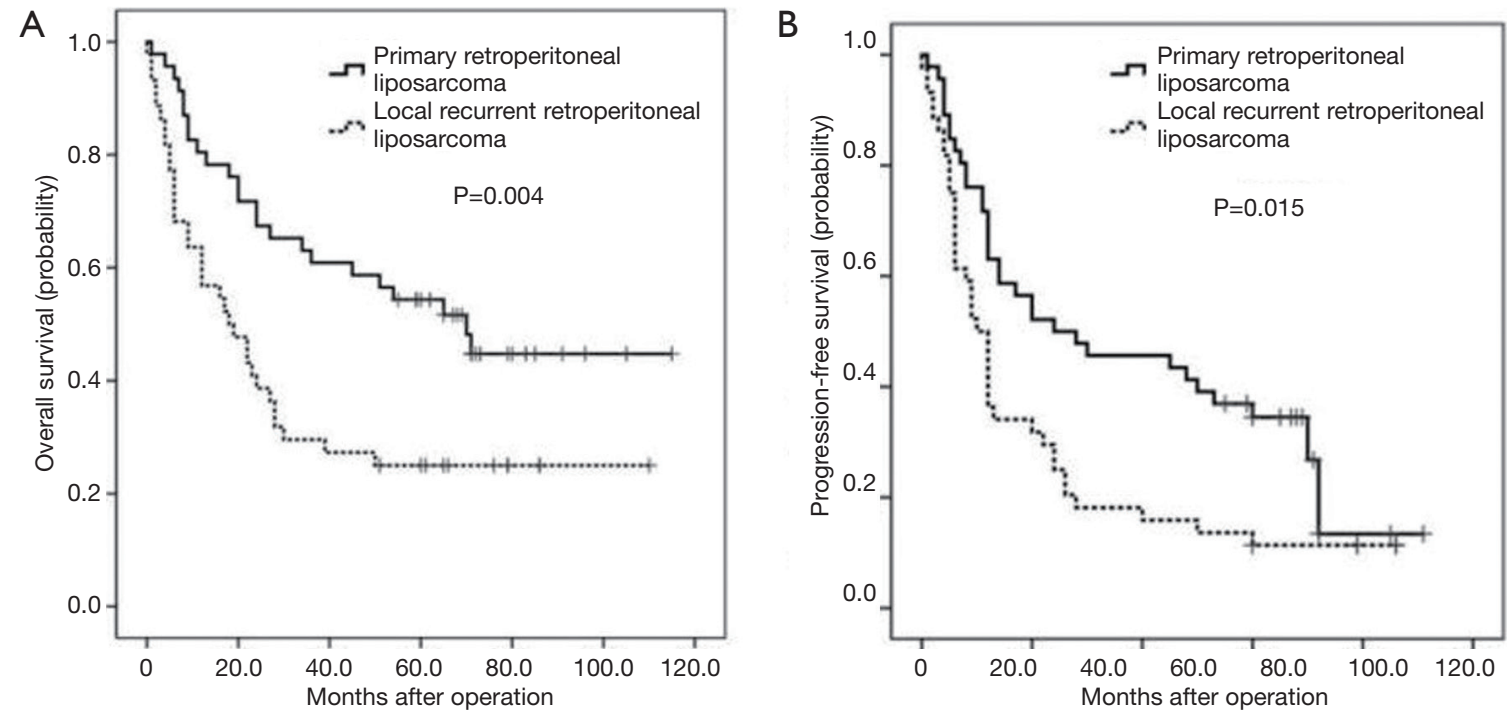

Figure 1 A postoperative survival curve for RPLPS patients stratified by referral status for primary tumor $v s$. local recurrent tumor cases. (A) OS after operation; (B) PFS after operation. RPLPS, retroperitoneal liposarcoma; OS, overall survival; PFS, progression-free survival.

complete resection rate compared to the relapsed group (R0, $34.1 \%$; R1, 40.9\%; R2, 25\%) (P=0.004). In order to obtain a higher rate of complete tumor resection or to reduce tumor burden, $65.6 \%$ (59/90) of patients underwent at least 1 adjacent organ resection during surgery (Table S1). The most frequent organ resection performed was for the colon $(32.2 \%)$, followed by the kidney (18.9\%), spleen $(10.0 \%)$, and small intestine $(7.8 \%)$. The median blood loss in the PR group was $800 \mathrm{~mL}$ (95\% CI: 897.7-1,541.3), and $1,200 \mathrm{~mL}$ (95\% CI: 1,127.1-2,811.6) in the LR group. Assigning 1,500 $\mathrm{mL}$ of blood volume as a boundary, the LR group was more prone to blood loss during the operation $(\mathrm{P}=0.009)$. In 16 patients, tumors were closely associated with abdominal blood vessels, including the iliac vessels $(11.1 \%)$, inferior vena cava $(8.9 \%)$, and abdominal aorta (5.6\%). Postoperative complications included hemorrhagic shock $(\mathrm{n}=3)$, postoperative abdominal infections (3 cases), pancreatic fistulas $(n=2)$, intestinal fistula $(n=1)$, ureteral fistula $(n=1)$, and incisional liquefaction $(n=2)$. Adjuvant therapy was performed on 15 participants (12 in the relapse group), where 5 were treated with intraoperative radiotherapy, 6 with radiofrequency ablation, and 4 with chemotherapy.

\section{Outcomes}

Median follow-up time was 28 months. A total of 59 patients who underwent R0/R1 resection showed recurrence at a rate of $77.6 \%$, with a median recurrence time of 12 months. A total of 57 patients died, including 43 who died of relapse after R0/R1 resection, and 14 patients who passed away after R2 resection. The OS at 1, 3, and 5 years were $68.9 \%$, $45.6 \%$, and $33.3 \%$, respectively. The PFS at 1,3 , and 5 years were $50.0 \%, 32.2 \%$, and $18.9 \%$, respectively. Due to a differential course of the disease, the OS in the PR group was $80.4 \%, 60.9 \%$, and $45.7 \%$ at 1,3 , and 5 years, respectively, while the OS in the LR group was $56.8 \%$, $29.5 \%$, and $20.5 \%$, respectively. Differences between the 2 groups were statistically significant $(\mathrm{P}=0.004$, Figure $1 A)$. Similarly, the PFS at 1,3 , and 5 years in the PR group were $63.0 \%, 43.5 \%$, and $28.3 \%$, compared to $36.4 \%, 15.9 \%$, and $9.1 \%$ in the LR group $(\mathrm{P}=0.015$, Figure $1 B)$.

Among the 46 cases diagnosed with PR RPLPS, univariate analysis (Table S2) revealed that $\mathrm{R} 0$ resection resulted in longer $\mathrm{OS}$ than $\mathrm{R} 1$ and $\mathrm{R} 2$ resections (R0 resection median OS, 65 months, 95\% CI: 50.02-69.52 months vs. R1 resection median OS, 19 months, 95\% CI: $13.53-$ 56.14 months vs. R2 resection median OS, 8 months, $95 \%$ CI: 1.24-20.09 months, $\mathrm{P}<0.001$. Similarly, the median PFS of patients in the R0 resection group was 53 months ( $95 \%$ CI: 36.77-58.06 months) and 12 months for the R1 resection group (7.18-38.0 months). Assigning 1,500 mL of blood loss as a limit, a large amount of intraoperative bleeding indicated poor prognosis (OS, $\mathrm{P}=0.006$; $\mathrm{PFS}, \mathrm{P}=0.008$ ). The FNCLCC subtype was also a significant indicator of the postoperative survival time. The median OS times 
observed for FNCLCC grades 1, 2, and 3 were 70 (58.2380.93), 66 (34.42-75.38), and 20 (13.28-37.54) months $(\mathrm{P}<0.001)$, respectively. The median PFS were 55 (37.1761.57), 36 (14.73-60.07), and 12 (8.51-33.14) months $(\mathrm{P}=0.022)$, respectively. Multivariate analysis using the Cox proportional hazards model revealed that blood loss (OS: $\mathrm{P}=0.003$, Figure $2 A$; PFS: $\mathrm{P}=0.035$, Figure $2 B$ ), and tumor grade (OS: $\mathrm{P}=0.000$, Figure $2 C$; PFS: $\mathrm{P}=0.027$, Figure $2 D$ ) were significant prognostic factors associated with the postoperative survival time. However, extent of resection was a prognostic factor associated with $\mathrm{OS}(\mathrm{P}=0.035$, Figure 2E), but not with PFS (Table 2).

Among the 44 cases diagnosed as LR RPLPS, univariate analysis (Table S3) showed that a $\mathrm{R} 0$ resection results in longer OS (median OS, 61 months, 95\% CI: 38.32 72.61 months), and PFS (median PFS, 26 months, 95\% CI: 20.44-53.29 months). Patients with tumor diameters $<20 \mathrm{~cm}$ showed longer OS than patients with tumor diameters $\geq 20 \mathrm{~cm}$ (median OS, 30 months, $95 \%$ CI: 25.27 51.97 months vs. 9 months, $95 \%$ CI: 8.80-32.59 months, $\mathrm{P}=0.024)$. Differences of PFS between the 2 groups were not statistically significant $(\mathrm{P}=0.077)$. Patients with lowgrade disease showed favorable OS and PFS compared to those with intermediate-grade and high-grade disease (median OS: 72 months, 95\% CI: 42.55-94.95 months vs. 23 months, 95\% CI: 9.00-45.40 months vs. 12 months, 95\% CI: 10.31-25.46 months, $\mathrm{P}=0.001$; median PFS: 60 months, 95\% CI: 25.56-79.44 months vs. 12 months, 95\% CI: $5.58-18.43$ months vs. 8.5 months, $95 \%$ CI: 7.00-21.30 months, $\mathrm{P}=0.005)$. Multivariate analysis using the Cox proportional hazards model revealed that resection extent was a significant prognostic factor associated with OS $(\mathrm{P}=0.001$, Figure $3 A)$, whereas tumor grade was associated with PFS ( $\mathrm{P}=0.044$, Figure 3B) (Table 3).

\section{Discussion}

A continuous challenge is posed by RPLPS for diagnosis, prediction of clinical behavior, and treatment for disease recurrence within the intra-abdominal and retroperitoneal spaces. Due to the low overall incidence of this disease and variability between patients, little research has been undertaken to investigate RPLS in the Asian population (4-6).

Most patients diagnosed with RPLS have no obvious clinical symptoms in the early stage, and do not show signs until the tumor has enlarged and is causing symptoms. The relationship between tumor size and postoperative survival time remains controversial. Makela et al. believe that RPLPS is difficult to completely surgically remove, which affects survival time after surgery (7). In this study, patients with a tumor diameter $\geq 20 \mathrm{~cm}$ accounted for $50 \%$ of all cases (45/90). In the PR RPLPS group, there was no significant correlation between tumor size and survival prognosis. In the LR RLPPS group, the OS of patients with a tumor diameter $\geq 20 \mathrm{~cm}$ was significantly shorter than in patients with small tumor burden $(\mathrm{P}=0.024)$. However, this was not an independent risk factor for prognosis by multivariate analysis.

Patients with locally recurrent RPLPS are managed similarly to patients with primary RPLPS. Examination of suspected local recurrence requires abdominal CT, and sometimes MRI. Abdominal CT is not only the main preoperative diagnostic method, but also provides a basis for preoperative tumor classification $(8,9)$. Examination with CT determines tumor invasion in surrounding organs, blood vessels, and other tissues, which is critical for pre-surgical analysis in order to properly perform R0 resection. In this study, the colon was the most commonly resected organ $(32.2 \%)$, followed by the kidney (18.9\%). We observed that combined organ resection did not directly affect survival after surgery, which is similar to what has been observed in previous work (10). The use of preoperative percutaneous biopsies in RPLPS patients is controversial $(11,12)$. In cases where the diagnosis is not clear or the tumor is unresectable, biopsies can be used to confirm diagnosis and determine the histological type, providing additional information for adjuvant therapy.

Lehnert et al. retrospectively analyzed 110 RSTS patients and found that intraoperative bleeding $>1,500 \mathrm{~mL}$ was an independent risk factor for poor postoperative survival (13). Luo et al. found that amongst retroperitoneal dedifferentiated liposarcoma (RP DDLPS) patients, higher levels of intraoperative bleeding predicted a poor survival prognosis [hazard ratio (HR) 3.302, 95\% CI: 1.231-8.858, $\mathrm{P}=0.018]$ (14). In our study, univariate analysis revealed that intraoperative bleeding $<1,500 \mathrm{~mL}$ indicated better OS and PFS, but the effect of multivariate analysis was not significant in PR RPLPS. Although resection of RPLPS was more difficult, and was associated with more bleeding $(\mathrm{P}=0.009)$, no significant correlation was found between the amount of bleeding and postoperative survival in LR RPLPS.

Generally, active surgical resection is recommended to ensure clean margins that can effectively improve OS for RSTS (15). However, for RPLPS cases, especially recurrent liposarcoma cases, the relationship between the 


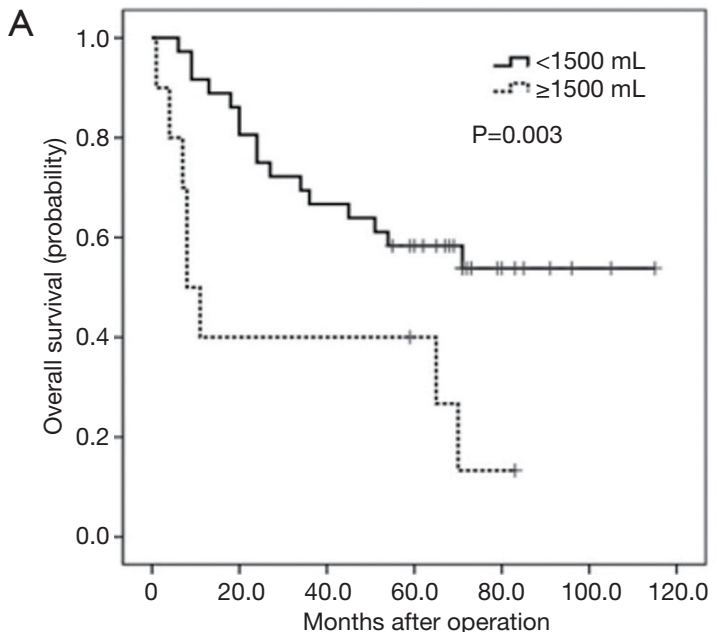

B

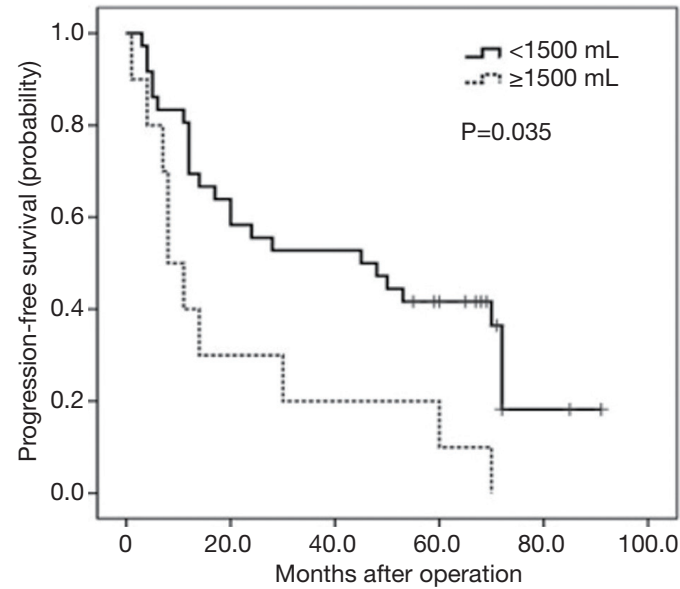

C
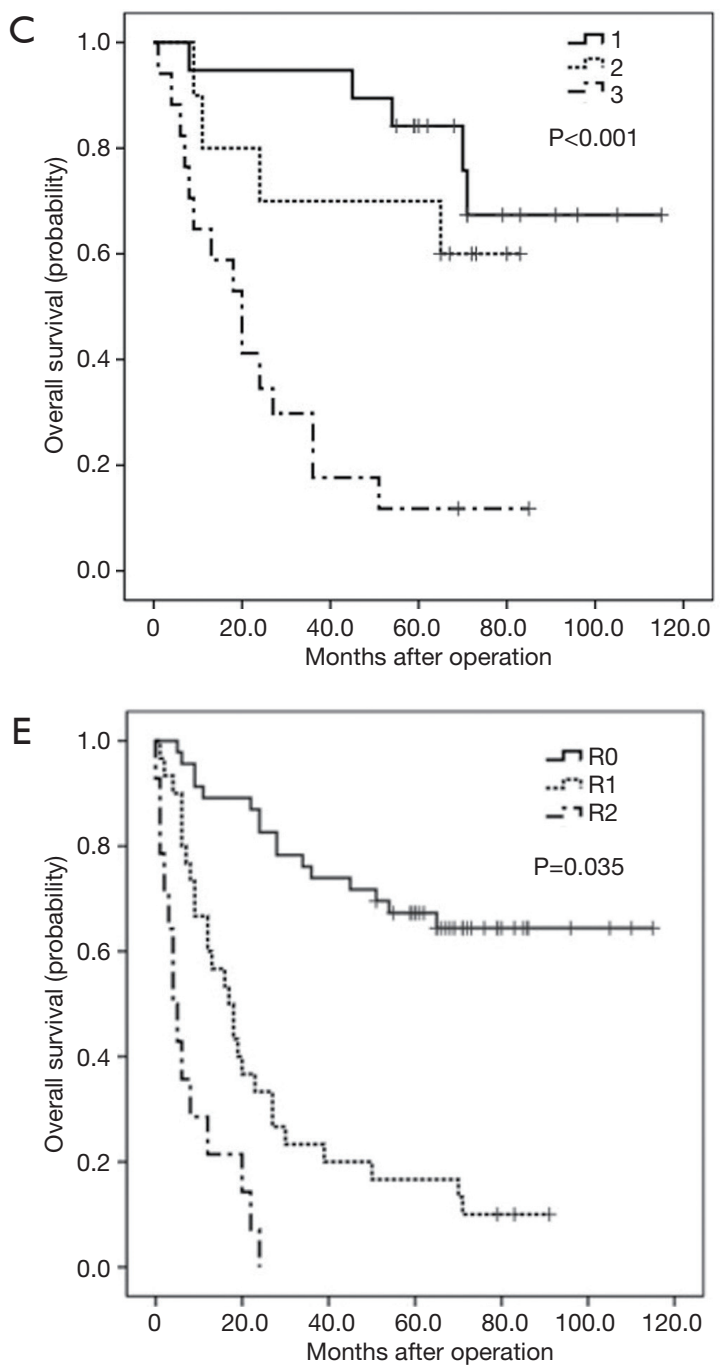

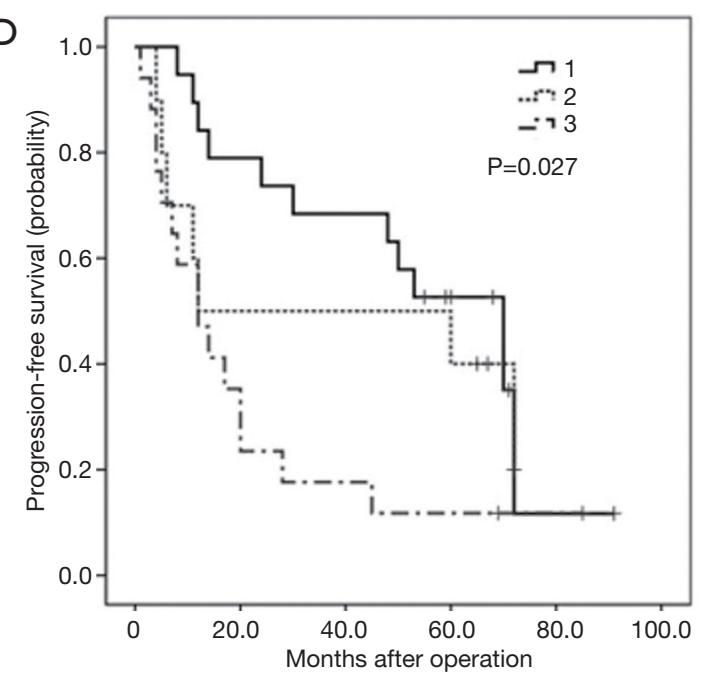

Figure 2 Resection outcomes for PR RPLPS cases according to blood loss [(A) OS; (B) PFS], tumor grade [(C) OS; (D) PFS] and resection margin status [(E) OS]. PR RPLPS, primary retroperitoneal liposarcoma; OS, overall survival; PFS, progression-free survival. 
Table 2 Multivariate associations between clinicopathologic and treatment factors and survival for PR RPLPS patients.

\begin{tabular}{|c|c|c|c|c|c|c|}
\hline \multirow{2}{*}{ Variable } & \multicolumn{6}{|c|}{ Analyses by survival type } \\
\hline & $\mathrm{HR}$ & $95 \% \mathrm{Cl}$ & $P$ value & HR & $95 \% \mathrm{Cl}$ & $P$ value \\
\hline Modus operandi (R0 vs. R1 vs. R2 resection) & & & $0.035^{\star}$ & & & 0.326 \\
\hline $\mathrm{R} 0$ vs. R1 & 3.02 & $0.96-9.48$ & 0.058 & 1.69 & $0.68-4.23$ & 0.260 \\
\hline Blood loss (<1,500 vs. $\geq 1,500 \mathrm{~mL})$ & 0.19 & $0.06-0.57$ & $0.003^{*}$ & 2.59 & $0.16-0.94$ & $0.035^{\star}$ \\
\hline FNCLCC (1 vs. 2 vs. 3) & & & $0.000^{*}$ & & & $0.027^{\star}$ \\
\hline 1 vs. 2 & 4.08 & $0.87-19.21$ & 0.075 & 1.57 & $0.57-4.29$ & 0.383 \\
\hline 1 vs. 3 & 17.68 & $4.70-66.56$ & $0.000^{\star}$ & 3.06 & $1.35-6.93$ & $0.007^{*}$ \\
\hline
\end{tabular}

*, significant. FNCLCC, French Federation of Cancer Centers Sarcoma Group; OS, overall survival; PFS, progression-free survival; R0 resection, Complete resection + clean microscopic margins; R1 resection, Complete resection + positive microscopic margins; R2 resection, macroscopically incomplete resection.
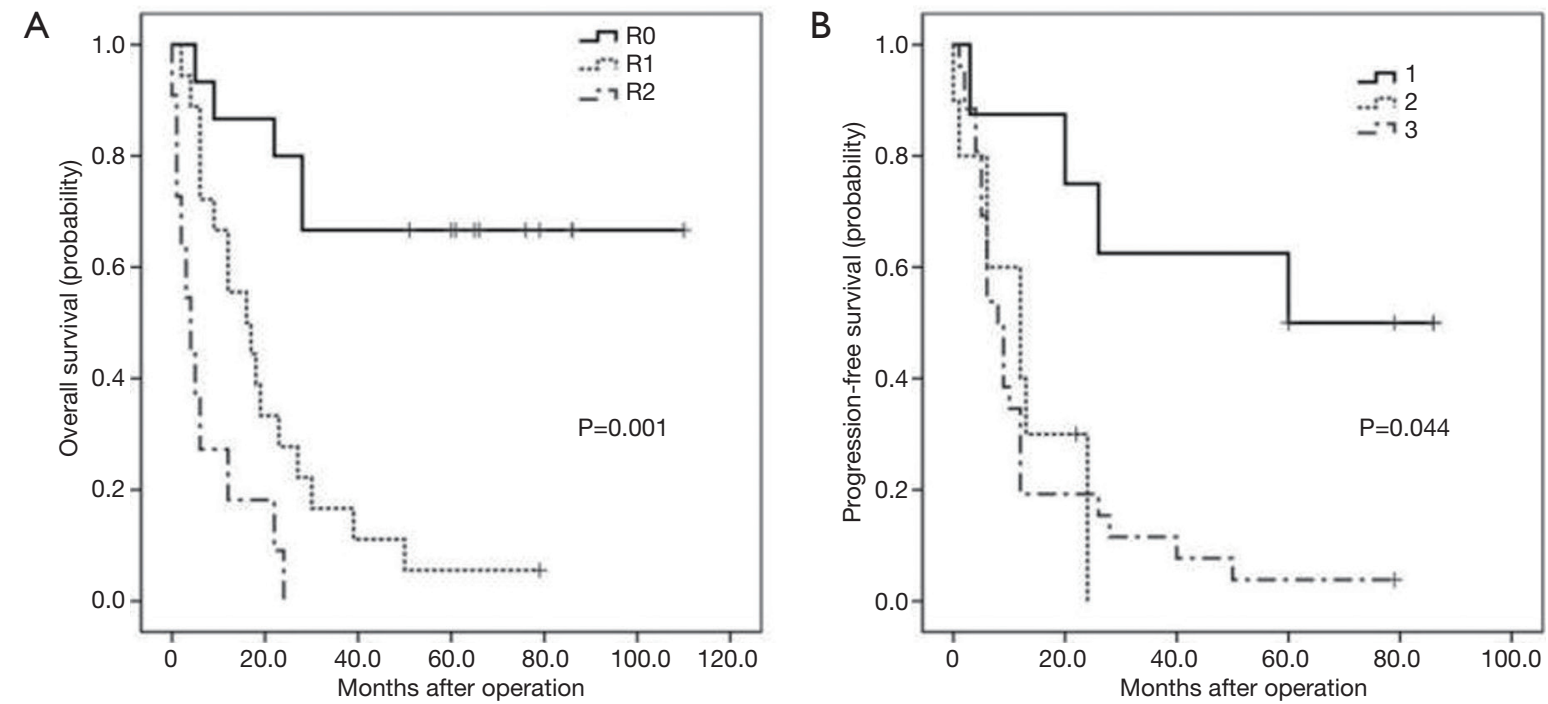

Figure 3 Resection outcomes for LR RPLPS cases according to resection margin status [(A) OS] and tumor grade [(B) PFS]. LR RPLPS, local recurrent retroperitoneal liposarcoma; OS, overall survival; PFS, progression-free survival.

marginal status and local recurrence is not clear. Zhao et al. suggest that a negative resection margin is associated with a reduction in local recurrence rate $(\mathrm{HR}=0.18, \mathrm{P}=0.001)$, and improved disease-specific survival ( $\mathrm{HR}=0.14, \mathrm{P}<0.001)(6)$. Keung et al. reported that 119 RP DDLPS cases showed no significant relationship between resection margin status and local recurrence (16). Univariate analysis of our study showed that surgical marginal status significantly affected OS and PFS, and multivariate analysis showed an R2 resection (as compared to an $\mathrm{R} 0$ resection) was associated with an increased risk for OS (PR RPLPS: HR 6.89, $\mathrm{P}=0.014$; LR RPLPS: HR 10.51, $\mathrm{P}=0.000$ ). The association of margin size on oncologic outcomes for RPLPS is based on limited data and varying opinions on the definition of a positive margin.

Previous studies $(17,18)$ have confirmed that histological grade is an important prognostic factor for retroperitoneal tumors, but relatively few studies have targeted liposarcomas, especially those that are recurrent. Zhao et al. suggest that low-grade RPLP tumors are significantly 
Table 3 Multivariate associations between clinicopathologic and treatment factors and survival for LR RPLPS patients.

\begin{tabular}{|c|c|c|c|c|c|c|}
\hline \multirow{2}{*}{ Variable } & \multicolumn{6}{|c|}{ Analyses by survival type } \\
\hline & $\mathrm{HR}$ & $95 \% \mathrm{Cl}$ & $P$ value & $\mathrm{HR}$ & $95 \% \mathrm{Cl}$ & $P$ value \\
\hline Modus operandi (R0 vs. R1 vs. R2 resection) & & & $0.001^{*}$ & & & 0.157 \\
\hline $\mathrm{R} 0$ vs. R1 & 3.94 & $1.32-11.78$ & $0.014^{*}$ & 2.21 & $0.99-4.94$ & 0.054 \\
\hline Tumor size (<20 vs. $\geq 20 \mathrm{~cm}$ ) & 0.49 & $0.22-1.09$ & 0.081 & 0.73 & $0.34-1.58$ & 0.422 \\
\hline FNCLCC (1 vs. 2 vs. 3) & & & 0.090 & & & $0.044^{*}$ \\
\hline 1 vs. 2 & 4.82 & $0.55-42.46$ & 0.157 & 5.58 & $1.29-24.14$ & $0.021^{*}$ \\
\hline 1 vs. 3 & 8.19 & $1.03-65.40$ & $0.047^{*}$ & 4.14 & $1.27-13.53$ & $0.019^{*}$ \\
\hline
\end{tabular}

*, significant. FNCLCC, French Federation of Cancer Centers Sarcoma Group; OS, overall survival; PFS, progression-free survival; R0 resection, Complete resection + clean microscopic margins; R1 resection, Complete resection + positive microscopic margins; R2 resection, macroscopically incomplete resection.

associated with a reduced risk of local recurrence and improved survival (6). Among the 119 cases of RP DDLPS studied by Keung et al., participants with intermediate grade RP DDLPS showed a better prognosis than those with high grade RP DDLPS (16). Altogether, these results support the FNCLCC classification (19), where differences between grade 1 and 3 tumors significantly affect OS and PFS, regardless of whether the tumors are primary or recurrent.

Due to the heterogeneity of tumor tissue, different tissue types often predict survival time $(2,20)$. A study by Gronchi et al. showed that myogenic differentiation significantly predicted the outcome of RPLPS (21). Despite that in this study, tumor histological type is not an independent risk factor for survival time after surgery, it is worth noting that transformation from retroperitoneal well-differentiated liposarcoma (RP WDLPS) to RP DDLPS occurs where there is an evolution of mutations following each recurrence (3). These mutations may also result in changes in tumor grade and affect patient survival. Therefore, the impact of different tumor histological types on the prognosis of RPLP requires further research.

Although surgery is considered the most effective treatment for RPLPS, some studies have also tried new adjuvant treatments. These studies have shown that preoperative neoadjuvant radiotherapy can prolong the survival of RPLPS, especially in patients with highrisk pathological types (22), and reduce the possibility of local recurrence (23). The MD Anderson Cancer Center reported that combined chemotherapy in patients with RP DDLPS produced a response rate of $24 \%$ (24). On account of new adjuvant treatment methods not having yet been widely clinically practiced, they are given to refractory or complicated liposarcoma cases. Thus, we did not discuss adjuvant treatment for the typical RPLPS case in our work.

In conclusion, we found that complete surgical resection is an independent favorable prognostic factor of OS for RPLPS (both primary and local recurrent). In PR RPLPS, intraoperative blood loss $\geq 1,500 \mathrm{~mL}$, and FNCLCC grade 3 predicted a poor prognosis (OS and PFS). Surgical resections of LR RPLPS are more difficult than PR RPLPS surgical resections, show more intraoperative blood loss, and worse pathological grade. Tumor grade is an independent risk factor for PFS in LR RPLPS. We anticipate that further prospective studies and multi-center collaborations will provide strong support for clinical decision-making for RPLPS treatment strategies.

\section{Acknowledgments}

We would like to thank all the investigators, surgeons, and patients who participated in this study.

Funding: This study was supported by the National Natural Science Foundation of China (NSFC 81871317) and the Technology Innovation Nursery Fund (16KMM15) of the Chinese PLA General Hospital.

\section{Footnote}

Reporting Checklist: The authors have completed the STROBE reporting checklist. Available at http://dx.doi. 
org/10.21037/atm-20-6316

Data Sharing Statement: Available at http://dx.doi. org/10.21037/atm-20-6316

Conflicts of Interest: All authors have completed the ICMJE uniform disclosure form (available at http://dx.doi. org/10.21037/atm-20-6316). The authors have no conflicts of interest to declare.

Ethical Statement: The authors are accountable for all aspects of the work in ensuring that questions related to the accuracy or integrity of any part of the work are appropriately investigated and resolved. The study was conducted in accordance with the Declaration of Helsinki (as revised in 2013). This study was approved by the Protection of Human Subjects Committee of the Chinese People's Liberation Army (PLA) General Hospital. Informed written consent was provided by all participants included in the study.

Open Access Statement: This is an Open Access article distributed in accordance with the Creative Commons Attribution-NonCommercial-NoDerivs 4.0 International License (CC BY-NC-ND 4.0), which permits the noncommercial replication and distribution of the article with the strict proviso that no changes or edits are made and the original work is properly cited (including links to both the formal publication through the relevant DOI and the license). See: https://creativecommons.org/licenses/by-nc-nd/4.0/.

\section{References}

1. Trans-Atlantic RPSWG. Management of Recurrent Retroperitoneal Sarcoma (RPS) in the Adult: A Consensus Approach from the Trans-Atlantic RPS Working Group. Ann Surg Oncol 2016;23:3531-40.

2. Gronchi A, Strauss DC, Miceli R, et al. Variability in Patterns of Recurrence After Resection of Primary Retroperitoneal Sarcoma (RPS): A Report on 1007 Patients From the Multi-institutional Collaborative RPS Working Group. Ann Surg 2016;263:1002-9.

3. Guo S, Xu Y, Qian F, et al. A recurrent giant retroperitoneal myxoid liposarcoma: a case report and literature review. Transl Cancer Res 2019;8:2672-6.

4. Neuhaus SJ, Barry P, Clark MA, et al. Surgical management of primary and recurrent retroperitoneal liposarcoma. Br J Surg 2005;92:246-52.
5. Lee SY, Goh BK, Teo MC, et al. Retroperitoneal liposarcomas: the experience of a tertiary Asian center. World J Surg Oncol 2011;9:12.

6. Zhao X, Li P, Huang X, et al. Prognostic factors predicting the postoperative survival period following treatment for primary retroperitoneal liposarcoma. Chin Med J (Engl) 2015;128:85-90.

7. Makela J, Kiviniemi H, Laitinen S. Prognostic factors predicting survival in the treatment of retroperitoneal sarcoma. Eur J Surg Oncol 2000;26:552-5.

8. Tateishi U, Hasegawa T, Beppu Y, Satake M, Moriyama N. Primary dedifferentiated liposarcoma of the retroperitoneum. Prognostic significance of computed tomography and magnetic resonance imaging features. J Comput Assist Tomogr 2003;27:799-804.

9. Lu J, Qin Q, Zhan LL, et al. Computed tomography manifestations of histologic subtypes of retroperitoneal liposarcoma. Asian Pac J Cancer Prev 2014;15:6041-6.

10. Tseng WW, Wang SC, Eichler CM, et al. Complete and safe resection of challenging retroperitoneal tumors: anticipation of multi-organ and major vascular resection and use of adjunct procedures. World J Surg Oncol 2011;9:143.

11. Ikoma N, Torres KE, Somaiah N, et al. Accuracy of preoperative percutaneous biopsy for the diagnosis of retroperitoneal liposarcoma subtypes. Ann Surg Oncol 2015;22:1068-72.

12. Raut CP, Pisters PW. Retroperitoneal sarcomas: Combined-modality treatment approaches. J Surg Oncol 2006;94:81-7.

13. Lehnert T, Cardona S, Hinz U, et al. Primary and locally recurrent retroperitoneal soft-tissue sarcoma: local control and survival. Eur J Surg Oncol 2009;35:986-93.

14. Luo P, Cai W, Yang L, et al. Retroperitoneal dedifferentiated liposarcoma: Analysis of 61 cases from a large institution. J Cancer 2018;9:3831-8.

15. Anaya DA, Lev DC, Pollock RE. The role of surgical margin status in retroperitoneal sarcoma. J Surg Oncol 2008;98:607-10.

16. Keung EZ, Hornick JL, Bertagnolli MM, et al. Predictors of outcomes in patients with primary retroperitoneal dedifferentiated liposarcoma undergoing surgery. J Am Coll Surg 2014;218:206-17.

17. Bonvalot S, Rivoire M, Castaing M, et al. Primary retroperitoneal sarcomas: a multivariate analysis of surgical factors associated with local control. J Clin Oncol 2009;27:31-7.

18. Gronchi A, Lo Vullo S, Fiore M, et al. Aggressive surgical 
policies in a retrospectively reviewed single-institution case series of retroperitoneal soft tissue sarcoma patients. J Clin Oncol 2009;27:24-30.

19. Matthyssens LE, Creytens D, Ceelen WP. Retroperitoneal liposarcoma: current insights in diagnosis and treatment. Front Surg 2015;2:4.

20. Singer S, Antonescu CR, Riedel E, et al. Histologic subtype and margin of resection predict pattern of recurrence and survival for retroperitoneal liposarcoma. Ann Surg 2003;238:358-70; discussion 370-1.

21. Gronchi A, Collini P, Miceli R, et al. Myogenic differentiation and histologic grading are major prognostic determinants in retroperitoneal liposarcoma. Am J Surg Pathol 2015;39:383-93.

Cite this article as: Yan Y, Xia S, Teng D, Hu S, Li S, Wang Y, Du X, Li R. Resection outcomes for primary and local recurrent retroperitoneal liposarcoma patients. Ann Transl Med 2020;8(21):1450. doi: 10.21037/atm-20-6316
22. Ecker BL, Peters MG, McMillan MT, et al. Preoperative radiotherapy in the management of retroperitoneal liposarcoma. Br J Surg 2016;103:1839-46.

23. Molina G, Hull MA, Chen YL, et al. Preoperative radiation therapy combined with radical surgical resection is associated with a lower rate of local recurrence when treating unifocal, primary retroperitoneal liposarcoma. J Surg Oncol 2016;114:814-20.

24. Livingston JA, Bugano D, Barbo A, et al. Role of chemotherapy in dedifferentiated liposarcoma of the retroperitoneum: defining the benefit and challenges of the standard. Sci Rep 2017;7:11836.

(English Language Editor: R. Scott) 